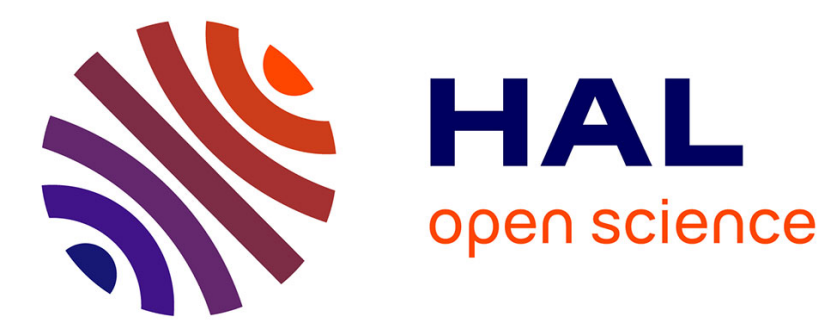

\title{
Spectral shaping of femtosecond pulses in aperiodic quasi-phase-matched gratings
}

Matteo Conforti, Fabio Baronio, Costantino De Angelis, Guido Sanna, Davide Pierleoni, Paolo Bassi

\section{- To cite this version:}

Matteo Conforti, Fabio Baronio, Costantino De Angelis, Guido Sanna, Davide Pierleoni, et al.. Spectral shaping of femtosecond pulses in aperiodic quasi-phase-matched gratings. Optics Communications, 2008, 281 (6), pp.1693-1697. 10.1016/j.optcom.2007.11.046 . hal-02397826

\section{HAL Id: hal-02397826 \\ https://hal.science/hal-02397826}

Submitted on 6 Dec 2019

HAL is a multi-disciplinary open access archive for the deposit and dissemination of scientific research documents, whether they are published or not. The documents may come from teaching and research institutions in France or abroad, or from public or private research centers.
L'archive ouverte pluridisciplinaire HAL, est destinée au dépôt et à la diffusion de documents scientifiques de niveau recherche, publiés ou non, émanant des établissements d'enseignement et de recherche français ou étrangers, des laboratoires publics ou privés. 


\title{
Spectral shaping of femtosecond pulses in aperiodic quasi-phase-matched gratings
}

\author{
Matteo Conforti, Fabio Baronio, Costantino De Angelis \\ Consorzio Nazionale Interuniversitario per le Scienze Fisiche della Materia, \\ Dipartimento di Elettronica per l'Automazione, Università di Brescia, Via Branze \\ 38, I 25123 Brescia, Italy \\ Guido Sanna, Davide Pierleoni, Paolo Bassi \\ Dipartimento di Elettronica Informatica e Sistemistica, Università di Bologna, \\ Viale Risorgimento 2, I 40136 Bologna, Italy
}

\begin{abstract}
We analyze the use of cascading second harmonic interactions in quadratic nonlinear crystals to mould the spectral characteristics of broadband near-infrared femtosecond pulses. Using a genetic algorithm, we optimize the design of the aperiodically poled ferroelectric crystal capable of generating the desired femtosecond infrared pulsed radiation.
\end{abstract}

Key words: Quasi phase matching, Second harmonic generation, Pulse shaping, Genetic algorithms

PACS: 42.65.Ky, 42.65.Re, 42.65.Wi, 42.70.Mp, 42.79.Nv, 02.60.Pn

\section{Introduction}

Important practical applications of high-energy femtosecond laser sources are emerging in a variety of fields, including material processing for optical [1] or mechanical [2] applications, biotechnology and medicine [3], environmental monitoring [4], and ultra-fast chemistry [5]. The development of compact and reliable sources with the desired temporal and spectral characteristics is then of current high technological interest.

In this framework, self-induced effects occurring during propagation of light in nonlinear media can be exploited to modify light propeties in the spatial, temporal and spectral domains [6]. Specifically, nonlinear spectral manipulation 
could be used to design devices enabling to modulate the amplitude, phase profile, and carrier frequency of femtosecond pulses over a broad range. These devices, potentially much simpler than optical parametric amplifiers [7], could enhance the performances of sources such as fibre lasers [8], which are very compact and efficient but do not offer frequency tunability.

In this context a key role is played by the choice of the nonlinear medium where self-induced effects take place: periodically poled optical crystals offer unique possibilities since they provide high effective nonlinearity and the flexibility of engineering the nonlinear poled structure with a photolithographic mask. Transverse quasi-phase-matched gratings have been made both for shaping of second harmonic beams [9] and to extend the spectral coverage of optical parametric oscillators [10]. Pulse compression, gain conversion [11,12] and spectral shift [13] enhancement in frequency-conversion schemes in longitudinally chirped quasi-phase-matched gratings have been demonstrated. More recently the issue of arbitrary second-harmonic pulse shaping in aperiodically poled crystals was solved by means of both optimal control and simulated annealing techniques [14-16].

In this Paper, we analyze the use of aperiodically poled nonlinear crystals to tune the carrier frequency and spectral profile of fundamental frequency (FF) broadband near-infrared femtosecond pulses, by means of cascading second harmonic ( $\mathrm{SH}$ ) interactions. To this goal, we use a genetic algorithm for properly designing piecewise uniform periodically poled ferroelectric crystals capable of moulding femtosecond infrared pulsed radiation. After briefly recalling the basic equations describing the phenomenon we are investigating and the features of genetic algorithms, we will report and comment results concerning some possible applications of the proposed structures.

\section{Aperiodic quasi-phase-matched gratings for spectral shaping}

We consider the process of parametric quadratic wave mixing in a non uniform quasi-phase-matching (QPM) structure with variable QPM period. Averaging over the QPM periods, at the lowest order the coupled nonlinear partial differential equations governing the propagation of the complex electric field amplitudes $E_{m}(z, t)(m=1$ fundamental field $\mathrm{FF}, m=2$ second harmonic field $\mathrm{SH})$ of two plane waves of central frequencies $\omega_{i}\left(\omega_{2}=2 \omega_{1}\right)$ and wave numbers $k_{m}$ travelling along the $z$ axis read:

$$
\begin{aligned}
& i \frac{\partial E_{1}}{\partial z}=\frac{\beta_{1}}{2} \frac{\partial^{2} E_{1}}{\partial t^{2}}-\chi_{1} \exp [-i \Delta k(z) z] E_{1}^{*} E_{2}, \\
& i \frac{\partial E_{2}}{\partial z}=i \delta \frac{\partial E_{2}}{\partial t}+\frac{\beta_{2}}{2} \frac{\partial^{2} E_{2}}{\partial t^{2}}-\chi_{2} \exp [i \Delta k(z) z] E_{1}^{2},
\end{aligned}
$$


where $\Delta k(z)=2 k_{1}-k_{2}+2 \pi / \Lambda(z)$ is the effective wave-number mismatch, $\Lambda(z)$ is the variable poling period, $\delta$ is the difference between the inverse group velocity at $\mathrm{FF}$ and $\mathrm{SH}, \beta_{1,2}$ is the group velocity dispersion at $\mathrm{FF}$ and $\mathrm{SH}$, and $\chi_{m}=2 \omega_{1} \chi^{(2)} / \pi c n_{m}$, being $\chi^{(2)}$ the second-order susceptibility. In the following, since the refractive indices at FF and $\mathrm{SH}$ are similar $\left(n_{1} \approx n_{2}\right)$, we assume $\chi_{1}=\chi_{2}=\chi$.

The problem of the spectral control of the FF field at output by means of mismatch engineering can be stated as follows: having fixed the FF wave at the input of the nonlinear crystal, determine the piecewise constant effective mismatch $\Delta k(z)$ that minimizes a cost (or fitness) function.

For example we may want to minimize the distance between the FF spectrum at output $\left[\hat{E}_{1}(z=L, \omega)\right]$ and the target $\left[\hat{E}_{1, \text { target }}(\omega)\right]$, where $\hat{E}_{1}(\omega)$ stands for the Fourier transform of $E_{1}(t)$. As a measure of the distance between the target and the FF wave at output, we can use the following fitness function:

$$
J=\int_{-\infty}^{+\infty}\left|\hat{E}_{1}(z=L, \omega)-\hat{E}_{1, \text { target }}(\omega)\right|^{2} d \omega
$$

Another case of interest is the maximization of the shift of the center of mass of the FF spectrum toward red or blue. In this case the fitness function reads

$$
J= \pm \frac{\int_{-\infty}^{+\infty} \omega\left|\hat{E}_{1}(z=L, \omega)\right|^{2} d \omega}{\int_{-\infty}^{+\infty}\left|\hat{E}_{1}(z=L, \omega)\right|^{2} d \omega}
$$

where the $+(-)$ sign imposes redshift (blueshift).

Once the mismatch function $\Delta k(z)$ is obtained, it is straightforward to determine the nonuniform aperiodic QPM structure that implements the desired $\Delta k(z)$, by using the formula $\Lambda(z)=2 \pi /\left[\Delta k(z)-2 k_{1}+k_{2}\right]$.

The goal here is thus to find the optimum $\Delta k(z)$ function. Searching among a collection of candidate solutions for a desired one is a very common problem and many different algorithms can be used for this kind of optimization (as the Dijkstra or the Floyd algorithms). In this framework genetic algorithms are a class of optimization procedures that apply principles of natural evolution to optimize a given objective function $[17,18]$, and are able to solve a variety of problems that are not well suited for standard optimization algorithms, including problems in which the objective function is discontinuous, nondifferentiable, stochastic, or highly nonlinear. Genetic algorithms were recently successfully used even for solving very complex optical design problems [19]. 
The genetic procedure that we used to find the optimal $\Delta k(z)$ can be summarized in the following iterative algorithm:

(1) Given a crystal of length $L$, choose a suitable number of uniform domains characterized by $\Delta k_{n}$, with $n=1, \ldots, N$. The variables to be optimized $\left(\Delta k_{n}\right)$ are stored in a vector which is usually called individual. Each individual represents an aperiodic structure.

(2) Randomly generate an initial population of $M$ individuals. In order to have a sufficiently broad population it is advisable to take $M \geq N$.

(3) Compute the fitness function $J(m)$ for each individual $m$ in the current population, and select parents based on their fitness. This steps requires to solve $M$ times Eqs. (1) (we employed a standard finite-difference fourth order Runge-Kutta method).

(4) Generate the new population from the parents. Children are produced either by making random changes to a single parent (mutation) or by combining the vector entries of a pair of parents (crossover).

(5) If a generated individual fits the target, or more generally it meets a predefined convergence criterion, the iterative procedure stops, otherwise it goes back to point 3 .

\section{Results and discussion}

In order to show the capabilities of our method to design devices able to perform spectral shaping of femtosecond infrared pulses, we consider Periodically Poled Stoichiometric Lithium Tantalate (PPSLT) crystals. For FF input pulses with center wavelength of $1500 \mathrm{~nm}$, the parameters to be used in Eqs. (1) are: $\chi_{0}=1.87 \cdot 10^{-5} V^{-1}, \delta=4 \cdot 10^{-10} \mathrm{~s} / \mathrm{m}, \beta_{1}=0.94 \cdot 10^{-25} \mathrm{~s}^{2} / \mathrm{m}$ and $\beta_{2}=3.7 \cdot 10^{-25} \mathrm{~s}^{2} / \mathrm{m}$.

As a first example we apply our design method to blue shifting spectral shaping. We consider a $L=2.5 \mathrm{~cm}$ long crystal, a gaussian transform limited FF input with peak intensity $I=20 \mathrm{GW} / \mathrm{cm}^{2}$ and spectral full width at half maximum intensity (FWHMI) of $55 \mathrm{~nm}$ (corresponding to a temporal FWHMI of $T_{F F}=60 \mathrm{fs}$ ) centered at $\lambda=1500 \mathrm{~nm}$. As a target we choose a blue shifted FF gaussian wave with the same spectral bandwidth centered at $\lambda=1460 \mathrm{~nm}$ (blue shifting) and a FF maximum depletion of $50 \%$. We divide the crystal in $N=10$ uniform domains, which turned out to be a sufficiently large number to obtain reasonable agreement with the target. Using a higher number of domains does not considerably improve the performances of the method. In all the examples we obtained convergence to the optimal solution after around 500 iterations (or generations) of the genetic algorithm. These runs requires a CPU time of a few hours (1-4) on a standard personal computer. 
The results of the genetic algorithm based design are shown in Fig. 1. In Fig. 1 (a) we show the piecewise constant effective mismatch $\Delta k(z)$ of the PPLST aperiodic grating poling period. Figure $1(\mathrm{~b})$ shows the FF spectral evolution in the $(\lambda, z)$ plane: it is clear that the frequency shifting mainly takes place in the first half of the crystal, whereas in the last part the spectral shape is modified to fit the gaussian target. Figure 1(c) shows the spectral intensity of input FF field (blue line) and output FF field (thick black line) compared to the FF target field (red circles): the agreement is very good. Finally Fig. 1(d) shows the FF input (blue line) and output (black line) temporal pulse profile. The temporal profile is very clean, almost symmetric and delayed of about $100 \mathrm{fs}$. This delay is caused partially by the spectral blueshift, which moves the FF pulse in a spectral region where the group velocity is decreased owing to dispersion, an partially induced by an effective steepening due to the dragging by the slower $\mathrm{SH}$ pulse [20].
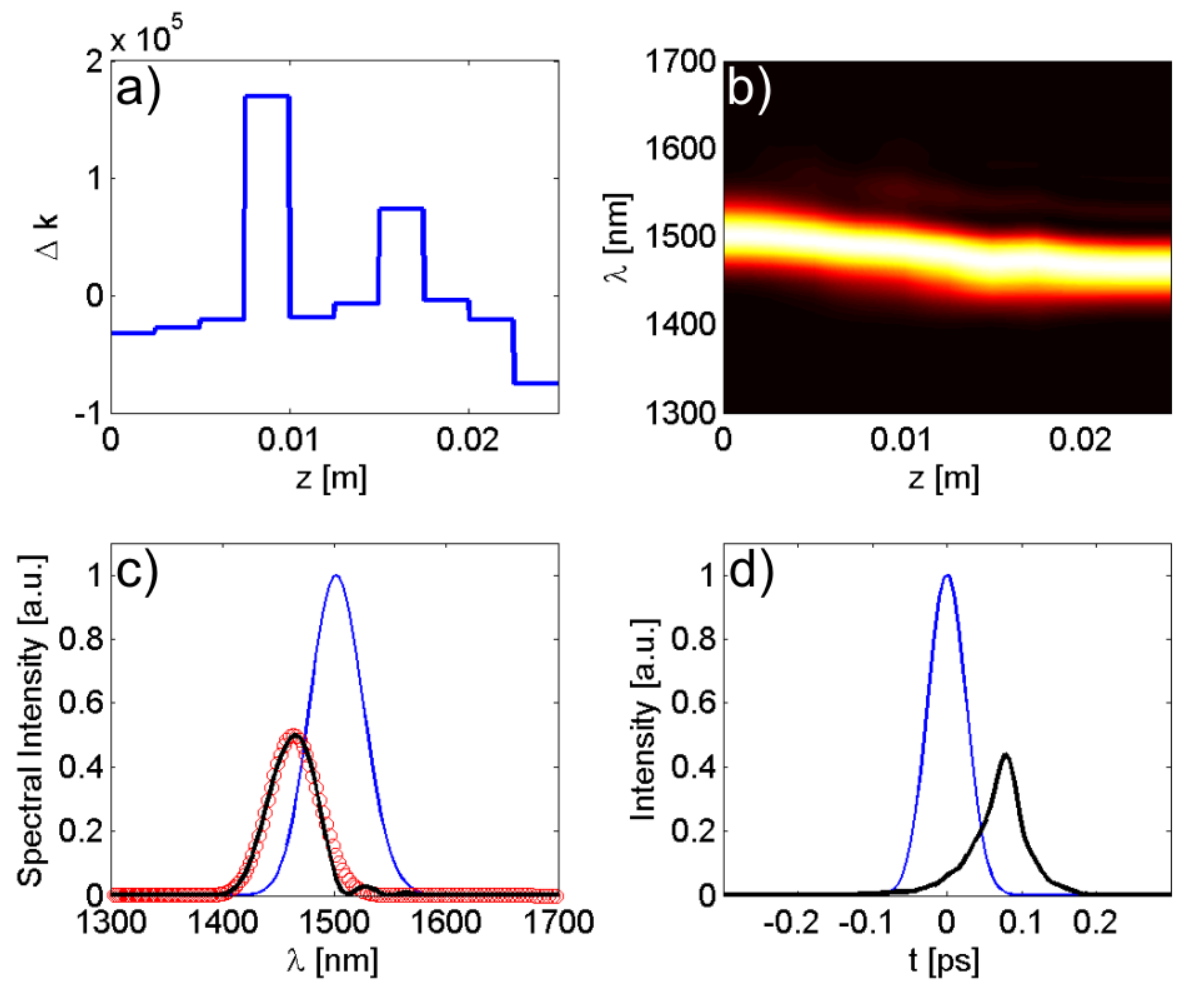

Fig. 1. (Color online) a) Effective mismatch profile $\Delta k(z)$. b) FF spectral evolution in the $(\lambda, z)$ plane. c) Spectral intensity profile of FF input field (thin blue line) and FF output field (thick black line) compared to the FF target (red circles). d) Input (thin blue line) and output (thick black line) FF temporal intensity profiles.

In the second example, using the same input signal, we apply our design procedure to red shifting spectral shaping. As a target we thus choose a FF gaussian wave of spectral full width at half maximum intensity (FWHMI) of $55 \mathrm{~nm}$ centered at $\lambda=1540 \mathrm{~nm}$ and a FF maximum depletion of $50 \%$. The results are shown in Fig. 2. In Fig. 2(a) we show the piecewise constant 
effective mismatch $\Delta k(z)$ of the PPLST grating. Figure 2(b) shows the FF spectral evolution in the $(\lambda, z)$ plane. Once again we can note that the spectral shift takes place mostly in the first half of the device, while the last part of the crystal engineers the spectral shape of the output. Figure 2(c) reports the spectral intensity of input FF field (thin blue line) and output FF field (thick black line) compared to the FF target field (red circles): the agreement is very good. Note that in this example the effective cascading nonlinearity is of self-focusing type [21], owing to the positive values of $\Delta k(z)$. This regime usually prevents to obtain clean spectral and temporal profiles, because of the combined effect of positive dispersion and effective self-focusing cascading nonlinearity, which eventually leads to pulse breakup. However the engineering of the grating prevents these undesirable phenomena and gives substantially good results. Finally Fig. 2(d) shows the FF (blue line) and output (black line) temporal intensity pulse profiles. The temporal profile is smooth and does not exhibit breakup phenomena.
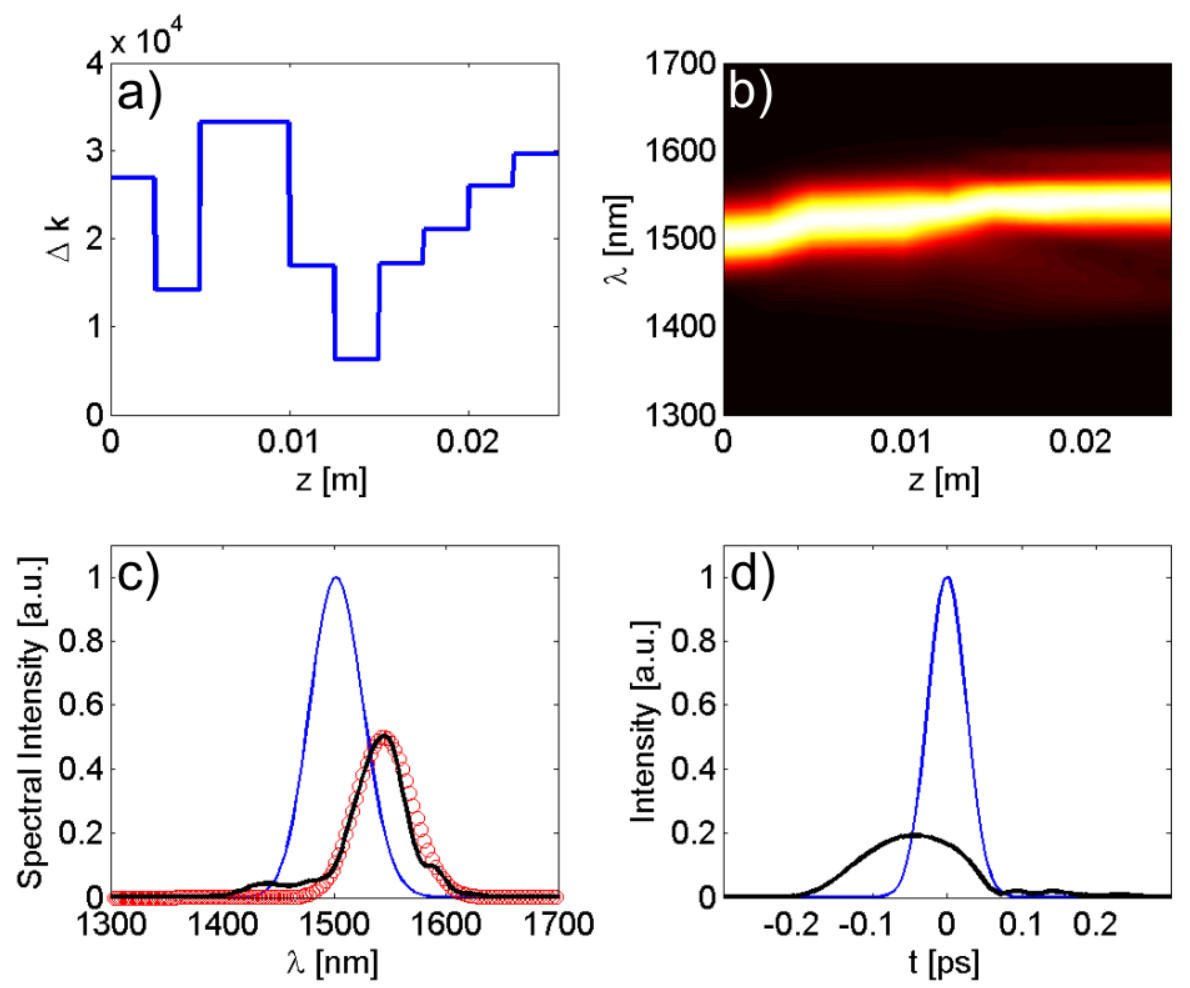

Fig. 2. (Color online) a) Effective mismatch profile $\Delta k(z)$. b) FF spectral evolution in the $(\lambda, z)$ plane. c) Spectral intensity profile of FF input field (blue line) and FF output field (black line) compared to the FF target (red circles). d) Input (blue line) and output (black line) FF temporal intensity profiles.

As demonstrated by the above examples, the spectral shift caused by a phasemismatched second harmonic generation process under conditions of strong group-velocity mismatch, can be efficiently controlled by acting on mismatch engineering. However spectral shifting can also be affected by other param- 
eters, mainly by the initial FF intensity and chirp [21]. Our genetic device design is well suited also to take into account these parameters. In the following we will present an example where the FF input chirp is among the accessible parameters to optimize in order to obtain the maximum spectral shift of the FF pulse. Thus the genetic variables to be optimized will include the mismatch $\Delta k(z)$ and the chirp $C$ of the input pulse, i.e. each individual in the genetic algorithm now represents an aperiodic structure and a particular chirp of the input pulse.

Let us now consider a $L=2.5 \mathrm{~cm}$ long crystal and a $\mathrm{FF}$ wave with a temporal gaussian profile with $T_{F F}=60 \mathrm{fs}$, input peak intensity $I=20 \mathrm{GW} / \mathrm{cm}^{2}$, centered at $\lambda=1500 \mathrm{~nm}$. As a target, we choose to maximize the spectral FF blue-shift by optimizing the mismatch grating and the initial FF pulse chirp using the fitness function (3).
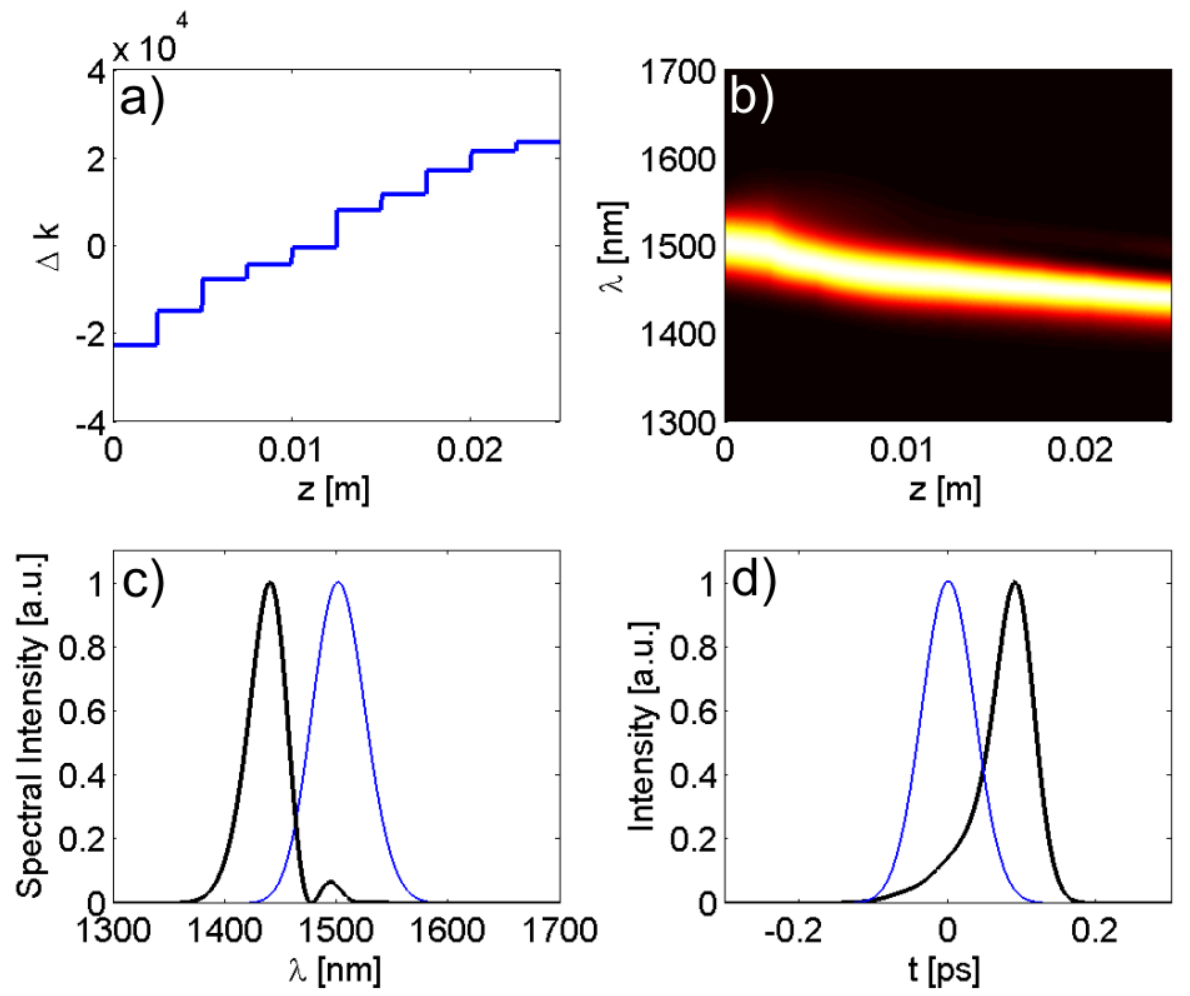

Fig. 3. (Color online) a) Effective mismatch profile $\Delta k(z)$. b) FF spectral evolution in the $(\lambda, z)$ plane. c) Spectral intensity profile of FF chirped input field (thin blue line) and normalized FF output field (thick black line). d) Input chirped (solid blue line) and normalized output (thick black line) FF temporal pulse profile.

The results of the iterative algorithm are shown in Fig. 3. In Fig. 3(a) we show the piecewise constant effective mismatch $\Delta k(z)$ of the PPLST grating. The values of $\Delta k(z)$ evolve in a linear fashion from $-20000 \mathrm{~m}^{-1}$ to $20000 \mathrm{~m}^{-1}$. A similar result was obtained in Ref. [13], where a linear variation of $\Delta k(z)$ was assumed. Figure 3(a) shows that the linear dependence is indeed the best 
choice and also identifies the best values of the initial and final mismatch. The optimized initial chirp is $C=-\beta_{1} z_{c}$ with $z_{c}=-0.013 \mathrm{~m}$ which means that the waist plane of the input FF gaussian pulse is roughly located in the middle of the crystal. Figure $3(\mathrm{~b})$ shows the FF spectral evolution in the $(\lambda, z)$ plane. Figure 3(c) reports the spectral intensity of chirped input FF field (thin blue line) and normalized output FF field (thick black line). Even if the target pulse shape is not imposed by the fitness function, the genetic algorithm gives a gaussian-shaped profile. The maximum attainable shift is $65 \mathrm{~nm}$, with a strong pump depletion (about 88\%). This strong pump depletion occurs since the values of the shift are related to energy exchange between $\mathrm{FF}$ and $\mathrm{SH}$ : as a rule of thumb the more energy is converted from $\mathrm{FF}$ to $\mathrm{SH}$, the higher is the frequency shift. Finally Fig. 3(d) shows the FF chirped input (solid blue line) and normalized FF output temporal pulse profile (thick black line). As clearly shown in the picture, by properly acting on the chirp of the input field it is possible to simultaneously control the output fundamental field spectral shape and to compensate dispersive effects to get an output temporal FWHMI smaller than the input one.

\section{Conclusions}

In conclusion a genetic technique has been used to mould the spectral properties of femtosecond infrared optical pulses, propagating in longitudinally aperiodic poled crystals, by mismatched second order parametric processes. In our work we account for the engineering of the non uniform QPM structure and the optimization of the input pulse characteristics (i.e., the initial chirp). Our results suggest a valuable means for shaping infrared femtosecond pulses in a way that is simpler and cheaper than the state of art. In fact the proposed devices are potentially much simpler than optical parametric amplifiers and could enhance the performances of sources such as fibre lasers, which are very compact and efficient but emit at a single fixed frequency. Moreover the method described here is quite general and can be exploited in other areas of optics whenever the spectral control of the optical pulse is required.

\section{Acknowledments}

Part of this work has been developed in the framework of the project PRIN 2005 "Spectral and temporal control of femtosecond pulses by second order non linear processes". 


\section{References}

[1] R. Osellame, V. Maselli, R. Martinez Vazquez, R. Ramponi and G. Cerullo, Appl. Phys. Lett. 90 (2007) 231118.

[2] J. H. Klein-Wiele J. Bekes and P. Pimon, Appl. Phys. A 79 (2004) 775.

[3] T. Juhasz, R. Kurtz, F. Raksi, C. Suarez, C. Horvath, and G. Spooner, Optics and Photonics News 13 (2002) 24.

[4] T. Fujii, N. Goto, M. Miki, T. Nayuki and K. Nemoto, Opt. Lett. 31 (2006) 3456 .

[5] C. Meier C and J. A. Beswick, J. Chem. Phys. 121 (2004), 4550.

[6] Y. S. Kivshar and G. P. Agrawal, Optical solitons, Academic press, San Diego, 2003.

[7] G. Cerullo and S. De Silvestri, Rev. Sci. Instrum. 74 (2003) 1.

[8] J. Limpert, T. Schreiber, S. Nolte, H. Zellmer, T. Tunnermann, R. Iliew, F. Lederer, J. Broeng, G. Vienne, A. Petersson, and C. Jakobsen, Opt. Express 11 (2003) 818.

[9] G. Imeshev, M. Proctor, and M. M. Feyer, Opt. Lett. 23 (1998) 673.

[10] P. E. Powers, T. J. Kulp, and S. E. Bisson, Opt. Lett. 23 (1998) 159.

[11] D. Artigas, D. T. Reid, M. M. Fejer and L. Torner, Opt. Lett. 27 (2002) 442.

[12] X. Zeng, S. Ashihara, N. Fujioka, T. Shimura, and K. Kuroda, Opt. Express 14 (2006) 9358.

[13] K. Beckwitt, F. O. Ilday, and F. W. Wise, Opt. Lett. 29 (2004) 763.

[14] M. Conforti, F. Baronio and C. De Angelis, Opt. Lett. 32 (2007) 1779.

[15] U. K. Sapaev and D. T. Reid, Opt. Epress 13 (2005) 3264.

[16] U. K. Sapaev and G. Assanto, Opt. Express 15 (2007) 7448.

[17] L. Davis, Genetic Algorithms and Simulated Annealing, Morgan Kaufmann, 1987.

[18] D. E. Goldberg, Genetic Algorithms in Search, Optimization and Machine Learning, Addison Wesley, 1987.

[19] J. Goh, I. Fushman, D. Englund, and J. Vuckovic, Opt. Express 13 (2007) 8218.

[20] M. Marangoni, C. Manzoni, R. Ramponi, G. Cerullo, F. Baronio, C. De Angelis and K. Kitamura, Opt. Lett. 31 (2006) 534.

[21] F. Baronio, C. De Angelis, M. Marangoni, C. Manzoni, R. Ramponi, and G. Cerullo, Opt. Express 14 (2006) 4774. 